\title{
On the Harary index of graph operations
}

\author{
Kinkar C Das ${ }^{1}$, Kexiang Xu ${ }^{2}$, Ismail Naci Cangul ${ }^{3 *}$, Ahmet Sinan Cevik ${ }^{4}$ and Ante Graovac ${ }^{5}$
}

"Correspondence:

cangul@uludag.edu.tr

${ }^{3}$ Department of Mathematics,

Faculty of Arts and Science, Uludag

University, Gorukle Campus, Bursa,

16059, Turkey

Full list of author information is

available at the end of the article

\begin{abstract}
The Harary index is defined as the sum of reciprocals of distances between all pairs of vertices of a connected graph. In this paper, expressions for the Harary indices of the join, corona product, Cartesian product, composition and disjunction of graphs are derived and the indices for some well-known graphs are evaluated. In derivations some terms appear which are similar to the Harary index and we name them the second and third Harary index.
\end{abstract}

MSC: 05C05; 05C07; 05C90

Keywords: graph; Harary index; graph operations

\section{Introduction and preliminaries}

Throughout this paper we consider simple connected graphs without loops and multiple edges. Suppose that $G$ is a graph with vertex set $V(G)=\left\{v_{1}, v_{2}, \ldots, v_{n}\right\}$ and edge set $E(G)$. The distance between the vertices $v_{i}$ and $v_{j}$ of $V(G)$ is denoted by $d_{G}\left(v_{i}, v_{j}\right)$ and it is defined as the number of edges in a minimal path connecting the vertices $v_{i}$ and $v_{j}$. The Harary index is one of very much studied topological indices and is defined as follows $[1,2]$ :

$$
H(G)=\sum_{v_{i}, v_{j} \in V(G), i \neq j} \frac{1}{d_{G}\left(v_{i}, v_{j}\right)}
$$

where the summation goes over all unordered pairs of vertices of $G$. Mathematical properties and applications of $H$ are reported in [3-11]. We now propose two more members of the class of Harary indices, the second Harary index and the third Harary index, which are as follows:

$$
H_{1}(G)=\sum_{v_{i}, v_{j} \in V(G), i \neq j} \frac{1}{d_{G}\left(v_{i}, v_{j}\right)+1}
$$

and

$$
H_{2}(G)=\sum_{v_{i}, v_{j} \in V(G), i \neq j} \frac{1}{d_{G}\left(v_{i}, v_{j}\right)+2},
$$

where the summation goes over all unordered pairs of the vertices of $G$. The Wiener index of $G$ is defined as [12]

$$
W=W(G)=\sum_{\left\{v_{i}, v_{j}\right\} \subseteq V} d_{G}\left(v_{i}, v_{j}\right) .
$$

(C) 2013 Das et al.; licensee Springer. This is an Open Access article distributed under the terms of the Creative Commons Attribution License (http://creativecommons.org/licenses/by/2.0), which permits unrestricted use, distribution, and reproduction in any medium, provided the original work is properly cited. 
If we denote by $d(G, k)$ the number of vertex pairs of $G$, the distance of which is equal to $k$, then the Wiener index of $G$ can be expressed as

$$
W(G)=\sum_{k \geq 1} k d(G, k)
$$

The maximum value of $k$, for which $d(G, k)$ is non-zero, is the diameter of the graph $G$, and it will be denoted by $D(G)$. The Wiener index is of certain importance in chemistry [13]. It is one of the oldest and most thoroughly studied graph-based molecular structuredescriptors (the so-called topological indices) [12-14]. Numerous chemical applications of it have been reported (see, for instance, $[15,16]$ ), and its mathematical properties are reasonably well understood [17-20]. The degree of $v_{i} \in V(G)$, denoted by $d_{G}\left(v_{i}\right)$, is the number of vertices in $G$ adjacent to $v_{i}$. For other undefined notations and terminology from graph theory, the readers are referred to [21].

In [22], Khalifeh et al. computed some exact formulae for the hyper-Wiener index of the join, Cartesian product, composition, disjunction and symmetric difference of graphs. Some more properties and applications of graph products can be seen in the classical book [23].

The paper is organized as follows. In Section 2, we obtain lower and upper bounds on the Harary index of graphs. In Section 3, we give some exact expressions for the Harary index of various graph operations, such as join, corona product, Cartesian product, composition, disjunction, etc. Moreover, computations are done for some well-known graphs.

\section{Bounds on the Harary index}

We define

$$
H_{t}(G)=\sum_{u_{i}, u_{k} \in V(G), i \neq k} \frac{1}{d_{G}\left(u_{i}, u_{k}\right)+t}
$$

where $t$ is any non-negative real number. In this section we obtain lower and upper bounds on $H_{t}(G)$ of the graph $G$. From that we can find lower and upper bounds on the Harary index of graphs. These results are useful in the next section. We begin with the following lower and upper bounds on $H_{t}(G)$.

Theorem 1 Let $G\left(\not K_{|G|}\right)$ be a connected graph of order $|G|,\|G\|$ edges and diameter $D(G)$. Then

$$
H_{t}(G) \geq \frac{\|G\|}{t+1}+\frac{\left(\frac{|G|(|G|-1)}{2}-\|G\|\right)^{2}}{W(G)+\frac{|G|(|G|-1)}{2} t-(t+1)\|G\|}
$$

with equality holding if and only if $G$ is isomorphic to a graph of diameter 2. Moreover,

$$
H_{t}(G) \leq \frac{\|G\|}{t+1}+\frac{\left(\frac{|G|(|G|-1)}{2}-\|G\|\right)\left[2+\left(\frac{|G|(|G|-1)}{2}-\|G\|-1\right) \times\left(\frac{D(G)+t}{t+2}+\frac{t+2}{D(G)+t}\right)\right]}{2\left(W(G)+\frac{|G|(|G|-1)}{2} t-(t+1)\|G\|\right)}
$$

with equality holding if and only if $G$ is isomorphic to a graph of diameter 2. 
Proof For $\left(u_{i}, u_{k}\right) \neq\left(u_{j}, u_{\ell}\right), i \neq k, j \neq \ell, 2 \leq d_{G}\left(u_{i}, u_{k}\right) \leq D(G), 2 \leq d_{G}\left(u_{j}, u_{\ell}\right) \leq D(G)$, we have

$$
2 \leq\left(\frac{d_{G}\left(u_{i}, u_{k}\right)+t}{d_{G}\left(u_{j}, u_{\ell}\right)+t}+\frac{d_{G}\left(u_{j}, u_{\ell}\right)+t}{d_{G}\left(u_{i}, u_{k}\right)+t}\right) \leq\left(\frac{D(G)+t}{t+2}+\frac{t+2}{D(G)+t}\right) .
$$

From the definition of the Wiener index, we have

$$
W(G)=\sum_{u_{i}, u_{k} \in V(G), i \neq k} d_{G}\left(u_{i}, u_{k}\right)
$$

Using the above, we get

$$
\sum_{u_{i}, u_{k} \in V(G), i \neq k, d_{G}\left(u_{i}, u_{k}\right) \geq 2}\left(d_{G}\left(u_{i}, u_{k}\right)+t\right)=W(G)+\frac{|G|(|G|-1)}{2} t-(t+1)\|G\| .
$$

Now,

$$
\begin{aligned}
& \sum_{u_{i}, u_{k} \in V(G), i \neq k, d_{G}\left(u_{i}, u_{k}\right) \geq 2}\left(d_{G}\left(u_{i}, u_{k}\right)+t\right) \sum_{u_{i}, u_{k} \in V(G), i \neq k, d_{G}\left(u_{i}, u_{k}\right) \geq 2} \frac{1}{d_{G}\left(u_{i}, u_{k}\right)+t} \\
= & \left(\frac{|G|(|G|-1)}{2}-\|G\|\right) \\
& +\sum_{\left(u_{i}, u_{k}\right) \neq\left(u_{j}, u_{\ell}\right), i \neq k, j \neq \ell, d_{G}\left(u_{i}, u_{k}\right) \geq 2, d_{G}\left(u_{j}, u_{\ell}\right) \geq 2}\left(\frac{d_{G}\left(u_{i}, u_{k}\right)+t}{d_{G}\left(u_{j}, u_{\ell}\right)+t}+\frac{d_{G}\left(u_{j}, u_{\ell}\right)+t}{d_{G}\left(u_{i}, u_{k}\right)+t}\right) .
\end{aligned}
$$

Using (3) in the above, we get

$$
\begin{aligned}
& \sum_{u_{i}, u_{k} \in V(G), i \neq k, d_{G}\left(u_{i}, u_{k}\right) \geq 2}\left(d_{G}\left(u_{i}, u_{k}\right)+t\right) \sum_{u_{i}, u_{k} \in V(G), i \neq k, d_{G}\left(u_{i}, u_{k}\right) \geq 2} \frac{1}{d_{G}\left(u_{i}, u_{k}\right)+t} \\
\geq & \left(\frac{|G|(|G|-1)}{2}-\|G\|\right)+\left(\frac{|G|(|G|-1)}{2}-\|G\|\right)\left(\frac{|G|(|G|-1)}{2}-\|G\|-1\right) \\
= & \left(\frac{|G|(|G|-1)}{2}-\|G\|\right)^{2}
\end{aligned}
$$

and

$$
\begin{aligned}
& \sum_{u_{i}, u_{k} \in V(G), i \neq k, d_{G}\left(u_{i}, u_{k}\right) \geq 2}\left(d_{G}\left(u_{i}, u_{k}\right)+t\right) \sum_{u_{i}, u_{k} \in V(G), i \neq k, d_{G}\left(u_{i}, u_{k}\right) \geq 2} \frac{1}{d_{G}\left(u_{i}, u_{k}\right)+t} \\
\leq & \left(\frac{|G|(|G|-1)}{2}-\|G\|\right)+\frac{\left(\frac{|G|(|G|-1)}{2}-\|G\|\right)\left(\frac{|G|(|G|-1)}{2}-\|G\|-1\right)}{2} \\
& \times\left(\frac{D(G)+t}{t+2}+\frac{t+2}{D(G)+t}\right) \text { by }(3) .
\end{aligned}
$$

Now,

$$
\begin{aligned}
H_{t}(G) & =\sum_{u_{i}, u_{k} \in V(G), i \neq k} \frac{1}{d_{G}\left(u_{i}, u_{k}\right)+t} \\
& =\frac{\|G\|}{t+1}+\sum_{u_{i}, u_{k} \in V(G), i \neq k, d_{G}\left(u_{i}, u_{k}\right) \geq 2} \frac{1}{d_{G}\left(u_{i}, u_{k}\right)+t} .
\end{aligned}
$$


Using (4), (6) and (7) in the above, we get the lower bound in (1) and upper bound in (2) on $H_{t}(G)$ of the graph $G$.

Now suppose that the equality holds in (1) and (2). Then all inequalities in the above argument must be equalities. For the lower bound, we must have

$$
d_{G}\left(u_{i}, u_{k}\right)=2 \text { for any pair of vertices }\left(u_{i}, u_{k}\right) \text { in } V(G) .
$$

For the upper bound, we must have

$$
d_{G}\left(u_{i}, u_{k}\right)=2=D(G) \quad \text { for any pair of vertices }\left(u_{i}, u_{k}\right) \text { in } V(G) \text {. }
$$

Thus $G$ is isomorphic to a graph of diameter 2 .

Conversely, one can see easily that both equalities hold in (1) and (2) for graphs of diameter 2 .

Corollary 1 Let $G\left(¥ K_{|G|}\right)$ be a connected graph of order $|G|,\|G\|$ edges and diameter $D(G)$. Then

$$
H_{1}(G) \geq \frac{\|G\|}{2}+\frac{\left(\frac{|G||| G \mid-1)}{2}-\|G\|\right)^{2}}{W(G)-2\|G\|+\frac{|G||G|-1)}{2}}
$$

with equality holding if and only if $G$ is isomorphic to a graph of diameter 2. Moreover,

$$
H_{1}(G) \leq \frac{\|G\|}{2}+\frac{\left(\frac{|G|(|G|-1)}{2}-\|G\|\right)\left[2+\left(\frac{|G|(|G|-1)}{2}-\|G\|-1\right) \times\left(\frac{D(G)+1}{3}+\frac{3}{D(G)+1}\right)\right]}{2\left(W(G)+\frac{|G|(|G|-1)}{2}-2\|G\|\right)}
$$

with equality holding if and only if $G$ is isomorphic to a graph of diameter 2.

Proof Putting $t=1$ in (1) and (2), we get the required result. By Theorem 1, we have that both equalities hold if and only if $G$ is isomorphic to a graph of diameter 2 .

Corollary 2 Let $G\left(¥ K_{|G|}\right)$ be a connected graph of order $|G|,\|G\|$ edges and diameter $D(G)$. Then

$$
H_{2}(G) \geq \frac{\|G\|}{3}+\frac{\left(\frac{|G|(|G|-1)}{2}-\|G\|\right)^{2}}{W(G)-3\|G\|+|G|(|G|-1)}
$$

with equality holding if and only if $G$ is isomorphic to a graph of diameter 2. Moreover,

$$
H_{2}(G) \leq \frac{\|G\|}{3}+\frac{\left(\frac{|G||| G \mid-1)}{2}-\|G\|\right)\left[2+\left(\frac{|G||| G \mid-1)}{2}-\|G\|-1\right) \times\left(\frac{D(G)+2}{4}+\frac{4}{D(G)+2}\right)\right]}{2(W(G)+|G|(|G|-1)-3\|G\|)}
$$

with equality holding if and only if $G$ is isomorphic to a graph of diameter 2.

Proof Putting $t=2$ in (1) and (2), we get the required result. By Theorem 1, we have that both equalities hold if and only if $G$ is isomorphic to a graph of diameter 2 .

From the above Theorem 1, we get the lower and upper bounds on the Harary index of graphs. 
Theorem 2 Let $G\left(\supsetneqq K_{|G|}\right)$ be a connected graph of order $|G|,\|G\|$ edges and diameter $D(G)$. Then

$$
H(G) \geq\|G\|+\frac{\left(\frac{|G||| G \mid-1)}{2}-\|G\|\right)^{2}}{W(G)-\|G\|}
$$

with equality holding if and only if $G$ is isomorphic to a graph of diameter 2. Moreover,

$$
H(G) \leq\|G\|+\frac{\left(\frac{|G||| G \mid-1)}{2}-\|G\|\right)\left[2+\left(\frac{|G||| G \mid-1)}{2}-\|G\|-1\right) \times\left(\frac{D(G)}{2}+\frac{2}{D(G)}\right)\right]}{2(W(G)-\|G\|)}
$$

with equality holding if and only if $G$ is isomorphic to a graph of diameter 2.

Proof Putting $t=0$ in (1) and (2), we get the required result. By Theorem 1, we have that both equalities hold if and only if $G$ is isomorphic to a graph of diameter 2 .

\section{Harary index of graph operations}

In this section, some exact formulae for the Harary index of some graph operations are presented.

Let $G_{1}$ and $G_{2}$ be two graphs with $\left|G_{1}\right|$ and $\left|G_{2}\right|$ vertices, and $\left\|G_{1}\right\|$ and $\left\|G_{1}\right\|$ edges, respectively. The join $G_{1} \vee G_{2}$ of graphs $G_{1}$ and $G_{2}$ with disjoint vertex sets $V\left(G_{1}\right)$ and $V\left(G_{2}\right)$, and edge sets $E\left(G_{1}\right)$ and $E\left(G_{2}\right)$ is the graph union $G_{1} \cup G_{2}$ together with all the edges joining $V\left(G_{1}\right)$ and $V\left(G_{2}\right)$. Thus, for example, $\bar{K}_{p} \vee \bar{K}_{q}=K_{p, q}$, the complete bipartite graph.

Theorem 3 Let $G_{1}$ and $G_{2}$ be two graphs. Then

$$
H\left(G_{1} \vee G_{2}\right)=\frac{1}{2}\left(\left|G_{1}\right|\left|G_{2}\right|+\left\|G_{1}\right\|+\left\|G_{2}\right\|\right)+\frac{1}{4}\left(\left|G_{1}\right|+\left|G_{2}\right|\right)\left(\left|G_{1}\right|+\left|G_{2}\right|-1\right) .
$$

Proof By the definition of the Harary index, we have

$$
\begin{aligned}
H\left(G_{1} \vee G_{2}\right)= & \sum_{u_{i}, v_{j} \in V\left(G_{1} \vee G_{2}\right), u_{i} \neq v_{j}} \frac{1}{d_{G_{1} \vee G_{2}}\left(u_{i}, v_{j}\right)} \\
= & \sum_{u_{i}, v_{j} \in V\left(G_{1}\right), u_{i} \neq v_{j}} \frac{1}{d_{G_{1} \vee G_{2}}\left(u_{i}, v_{j}\right)}+\sum_{u_{i}, v_{j} \in V\left(G_{2}\right), u_{i} \neq v_{j}} \frac{1}{d_{G_{1} \vee G_{2}}\left(u_{i}, v_{j}\right)} \\
& +\sum_{u_{i} \in V\left(G_{1}\right)} \sum_{v_{j} \in V\left(G_{2}\right)} \frac{1}{d_{G_{1} \vee G_{2}}\left(u_{i}, v_{j}\right)} \\
= & \frac{1}{2} \sum_{u_{i} \in V\left(G_{1}\right)}\left(d_{G_{1}}\left(u_{i}\right)+\frac{\left|G_{1}\right|-d_{G_{1}}\left(u_{i}\right)-1}{2}\right) \\
& +\frac{1}{2} \sum_{v_{j} \in V\left(G_{2}\right)}\left(d_{G_{2}}\left(v_{j}\right)+\frac{\left|G_{2}\right|-d_{G_{2}}\left(v_{j}\right)-1}{2}\right)+\left|G_{1}\right|\left|G_{2}\right| \\
& \text { as } d_{G_{1} \vee G_{2}}\left(u_{i}, v_{j}\right)= \begin{cases}0 & \text { if } u_{i}=v_{j}, \\
1 & \text { if } u_{i} v_{j} \in E\left(G_{1} \vee G_{2}\right), \\
2 & \text { otherwise }\end{cases}
\end{aligned}
$$




$$
\begin{aligned}
= & \frac{1}{2}\left(\left|G_{1}\right|\left|G_{2}\right|+\left\|G_{1}\right\|+\left\|G_{2}\right\|\right)+\frac{1}{4}\left(\left|G_{1}\right|+\left|G_{2}\right|\right)\left(\left|G_{1}\right|+\left|G_{2}\right|-1\right) \\
& \text { as } \sum_{u_{i} \in V\left(G_{1}\right)} d_{G_{1}}\left(u_{i}\right)=2\left\|G_{1}\right\| \text { and } \sum_{v_{j} \in V\left(G_{2}\right)} d_{G_{2}}\left(v_{j}\right)=2\left\|G_{2}\right\| .
\end{aligned}
$$

For two cycles $C_{m}$ and $C_{n}$, we have the following.

\section{Example 1}

$$
\begin{aligned}
H\left(C_{m} \vee C_{n}\right) & =\frac{1}{2}(m n+m+n)+\frac{1}{4}(m+n)(m+n-1) \\
& =\frac{1}{4}\left(m^{2}+n^{2}\right)+m n+\frac{1}{4}(m+n) .
\end{aligned}
$$

The corona product $G_{1} \circ G_{2}$ of two graphs $G_{1}$ and $G_{2}$ is defined to be the graph $\Gamma$ obtained by taking one copy of $G_{1}$ (which has $\left|G_{1}\right|$ vertices) and $\left|G_{1}\right|$ copies of $G_{2}$, and then joining the $i$ th vertex of $G_{1}$ to every vertex in the $i$ th copy of $G_{2}, i=1,2, \ldots,\left|G_{1}\right|$. Let $G_{1}=$ $(V, E)$ and $G_{2}=(V, E)$ be two graphs such that $V\left(G_{1}\right)=\left\{u_{1}, u_{2}, \ldots, u_{p_{1}}\right\}, p_{1}=\left|G_{1}\right|,\left|E\left(G_{1}\right)\right|=$ $\left\|G_{1}\right\|$ and $V\left(G_{2}\right)=\left\{v_{1}, v_{2}, \ldots, v_{p_{2}}\right\}, p_{2}=\left|G_{2}\right|,\left|E\left(G_{2}\right)\right|=\left\|G_{2}\right\|$. Then it follows from the definition of the corona that $G_{1} \circ G_{2}$ has $\left|G_{1}\right|\left(1+\left|G_{2}\right|\right)$ vertices and $\left\|G_{1}\right\|+\left|G_{1}\right|\left\|G_{2}\right\|+\left|G_{1}\right|\left|G_{2}\right|$ edges, where

$$
\begin{aligned}
V\left(G_{1} \circ G_{2}\right)= & \left\{\left(u_{i}, v_{j}\right), i=1,2, \ldots,\left|G_{1}\right| ; j=0,1,2 \ldots,\left|G_{2}\right|\right\} \text { and } \\
E\left(G_{1} \circ G_{2}\right)= & \left\{\left(\left(u_{i}, v_{0}\right),\left(u_{k}, v_{0}\right)\right),\left(u_{i}, u_{k}\right) \in E\left(G_{1}\right)\right\} \\
& \cup\left\{\left(\left(u_{i}, v_{j}\right),\left(u_{i}, v_{\ell}\right)\right),\left(v_{j}, v_{\ell}\right) \in E\left(G_{2}\right), i=1,2, \ldots,\left|G_{1}\right|\right\} \\
& \cup\left\{\left(\left(u_{i}, v_{0}\right),\left(u_{i}, v_{\ell}\right)\right), \ell=1,2, \ldots,\left|G_{2}\right|, i=1,2, \ldots,\left|G_{1}\right|\right\} .
\end{aligned}
$$

It is clear that if $G_{1}$ is connected, then $G_{1} \circ G_{2}$ is connected, and in general $G_{1} \circ G_{2}$ is not isomorphic to $G_{2} \circ G_{1}$.

Theorem 4 The Harary index of the corona product is computed as follows:

$$
H\left(G_{1} \circ G_{2}\right)=H\left(G_{1}\right)+\left|G_{2}\right| H_{1}\left(G_{1}\right)+\left|G_{2}\right|^{2} H_{2}\left(G_{1}\right)+\frac{1}{4}\left(\left|G_{2}\right|+3\right)\left|G_{1}\right|\left|G_{2}\right|+\frac{1}{2}\left|G_{1}\right|\left\|G_{2}\right\|,
$$

where $H_{1}\left(G_{1}\right)=\sum_{u_{i}, u_{k} \in V\left(G_{1}\right), u_{i} \neq u_{k}} \frac{1}{d_{G_{1}}\left(u_{i}, u_{k}\right)+1}$ and $H_{2}\left(G_{1}\right)=\sum_{u_{i}, u_{k} \in V\left(G_{1}\right), u_{i} \neq u_{k}} \frac{1}{d_{G_{1}}\left(u_{i}, u_{k}\right)+2}$.

Proof Note that

$$
H_{k}\left(G_{1}\right)=\sum_{u_{i}, u_{j} \in V\left(G_{1}\right), u_{i} \neq u_{j}} \frac{1}{d_{G_{1}}\left(u_{i}, u_{j}\right)+k} \quad \text { for } k=1,2 .
$$

By the definition of the Harary index, we have

$$
\begin{aligned}
H\left(G_{1} \circ G_{2}\right) & =\sum_{\left(u_{i}, v_{j}\right),\left(u_{k}, v_{\ell}\right) \in V\left(G_{1} \circ G_{2}\right),\left(u_{i}, v_{j}\right) \neq\left(u_{k}, v_{\ell}\right)} \frac{1}{d_{G_{1} \circ G_{2}}\left(\left(u_{i}, v_{j}\right),\left(u_{k}, v_{\ell}\right)\right)} \\
& =\sum_{\left(u_{i}, v_{0}\right),\left(u_{k}, v_{0}\right) \in V\left(G_{1} \circ G_{2}\right), i \neq k} \frac{1}{d_{G_{1} \circ G_{2}}\left(\left(u_{i}, v_{0}\right),\left(u_{k}, v_{0}\right)\right)}
\end{aligned}
$$




$$
\begin{aligned}
& +\sum_{\left(u_{i}, v_{0}\right),\left(u_{k}, v_{\ell}\right) \in V\left(G_{1} \circ G_{2}\right), \ell \neq 0} \frac{1}{d_{G_{1} \circ G_{2}}\left(\left(u_{i}, v_{0}\right),\left(u_{k}, v_{\ell}\right)\right)} \\
& +\sum_{\left(u_{i}, v_{j}\right),\left(u_{k}, v_{\ell}\right) \in V\left(G_{1} \circ G_{2}\right),\left(u_{i}, v_{j}\right) \neq\left(u_{k}, v_{\ell}\right), j \neq 0 \neq \ell} \frac{1}{d_{G_{1} \circ G_{2}}\left(\left(u_{i}, v_{j}\right),\left(u_{k}, v_{\ell}\right)\right)} \\
& =\sum_{u_{i}, u_{k} \in V\left(G_{1}\right), i \neq k} \frac{1}{d_{G_{1}}\left(u_{i}, u_{k}\right)} \\
& +\sum_{\left(u_{i}, v_{0}\right),\left(u_{i}, v_{\ell}\right) \in V\left(G_{1} \circ G_{2}\right), \ell \neq 0} \frac{1}{d_{G_{1} \circ G_{2}}\left(\left(u_{i}, v_{0}\right),\left(u_{i}, v_{\ell}\right)\right)} \\
& +\sum_{\left(u_{i}, v_{0}\right),\left(u_{k}, v_{\ell}\right) \in V\left(G_{1} \circ G_{2}\right), \ell \neq 0, i \neq k} \frac{1}{d_{G_{1} \circ G_{2}}\left(\left(u_{i}, v_{0}\right),\left(u_{k}, v_{\ell}\right)\right)} \\
& +\sum_{\left(u_{i}, v_{j}\right),\left(u_{i}, v_{\ell}\right) \in V\left(G_{1} \circ G_{2}\right), 0 \neq j \neq \ell \neq \neq 0} \frac{1}{d_{G_{1} \circ G_{2}}\left(\left(u_{i}, v_{j}\right),\left(u_{i}, v_{\ell}\right)\right)} \\
& +\sum_{\left(u_{i}, v_{j}\right),\left(u_{k}, v_{\ell}\right) \in V\left(G_{1} \circ G_{2}\right), j \neq 0 \neq \ell, i \neq k} \frac{1}{d_{G_{1} \circ G_{2}}\left(\left(u_{i}, v_{j}\right),\left(u_{k}, v_{\ell}\right)\right)} \\
& =H\left(G_{1}\right)+\sum_{u_{i} \in V\left(G_{1}\right)}\left|G_{2}\right|+\sum_{v_{j} \in V\left(G_{2}\right)} \sum_{u_{i}, u_{k} \in V\left(G_{1}\right), i \neq k} \frac{1}{d_{G_{1}}\left(u_{i}, u_{k}\right)+1} \\
& +\sum_{u_{i} \in V\left(G_{1}\right)} \frac{1}{2} \sum_{j=1}^{\left|G_{2}\right|}\left[d_{G_{2}}\left(v_{j}\right)+\frac{1}{2}\left(\left|G_{2}\right|-d_{G_{2}}\left(v_{j}\right)-1\right)\right] \\
& +\sum_{v_{j} \in V\left(G_{2}\right)} \sum_{u_{i}, u_{k} \in V\left(G_{1}\right), i \neq k} \frac{\left|G_{2}\right|}{d_{G_{1}}\left(u_{i}, u_{k}\right)+2} \\
& =H\left(G_{1}\right)+\left|G_{1}\right|\left|G_{2}\right|+\left|G_{2}\right| \sum_{u_{i}, u_{k} \in V\left(G_{1}\right), i \neq k} \frac{1}{d_{G_{1}}\left(u_{i}, u_{k}\right)+1} \\
& +\frac{1}{4}\left|G_{1}\right| \sum_{j=1}^{\left|G_{2}\right|}\left(\left|G_{2}\right|+d_{G_{2}}\left(v_{j}\right)-1\right)+\left|G_{2}\right|^{2} \sum_{u_{i}, u_{k} \in V\left(G_{1}\right), i \neq k} \frac{1}{d_{G_{1}}\left(u_{i}, u_{k}\right)+2} \\
& =H\left(G_{1}\right)+\left|G_{2}\right| H_{1}\left(G_{1}\right)+\left|G_{2}\right|^{2} H_{2}\left(G_{1}\right) \\
& +\frac{1}{4}\left(\left|G_{2}\right|+3\right)\left|G_{1}\right|\left|G_{2}\right|+\frac{1}{2}\left|G_{1}\right||| G_{2} \| .
\end{aligned}
$$

Theorem 5 Let $G_{1}\left(¥ K_{\left|G_{1}\right|}\right)$ and $G_{2}$ be two connected graphs with diameter of $G_{1}, D\left(G_{1}\right)$. Then the lower and upper bounds on the Harary index of the corona product are as follows:

$$
\begin{aligned}
& H\left(G_{1} \circ G_{2}\right) \\
& \geq\left[\frac{1}{W\left(G_{1}\right)-\left\|G_{1}\right\|}+\frac{\left|G_{2}\right|}{W\left(G_{1}\right)-2\left\|G_{1}\right\|+\frac{\left|G_{1}\right|\left(\left|G_{1}\right|-1\right)}{2}}\right. \\
& \left.\quad+\frac{\left|G_{2}\right|^{2}}{W\left(G_{1}\right)-3\left\|G_{1}\right\|+\left|G_{1}\right|\left(\left|G_{1}\right|-1\right)}\right] \\
& \quad \times\left(\frac{\left|G_{1}\right|\left(\left|G_{1}\right|-1\right)}{2}-\left\|G_{1}\right\|\right)^{2}+\left\|G_{1}\right\|\left(1+\frac{\left|G_{2}\right|}{2}+\frac{\left|G_{2}\right|^{2}}{3}\right) \\
& \quad+\frac{1}{4}\left(\left|G_{2}\right|+3\right)\left|G_{1}\right|\left|G_{2}\right|+\frac{1}{2}\left|G_{1}\right|\left\|G_{2}\right\|
\end{aligned}
$$


with equality holding if and only if $G_{1}$ is isomorphic to a graph of diameter 2. Moreover,

$$
\begin{aligned}
& H\left(G_{1} \circ G_{2}\right) \\
& \leq\left(\frac{\left|G_{1}\right|\left(\left|G_{1}\right|-1\right)}{2}-\left\|G_{1}\right\|\right) \times\left[\frac{2+\left(\frac{\left|G_{1}\right|\left(\left|G_{1}\right|-1\right)}{2}-\left\|G_{1}\right\|-1\right)\left(\frac{D\left(G_{1}\right)}{2}+\frac{2}{D\left(G_{1}\right)}\right)}{2\left(W\left(G_{1}\right)-\left\|G_{1}\right\|\right)}\right. \\
& \quad+\frac{\left|G_{2}\right|\left(2+\left(\frac{\left|G_{1}\right|\left(\left|G_{1}\right|-1\right)}{2}-\left\|G_{1}\right\|-1\right)\left(\frac{D\left(G_{1}\right)+1}{3}+\frac{3}{D\left(G_{1}\right)+1}\right)\right)}{2\left(W\left(G_{1}\right)-2\left\|G_{1}\right\|+\frac{\left|G_{1}\right|\left(\left|G_{1}\right|-1\right)}{2}\right)} \\
& \left.\quad+\frac{\left|G_{2}\right|^{2}\left(2+\left(\frac{\left|G_{1}\right|\left(\left|G_{1}\right|-1\right)}{2}-\left\|G_{1}\right\|-1\right)\left(\frac{D\left(G_{1}\right)+2}{4}+\frac{4}{D\left(G_{1}\right)+2}\right)\right)}{2\left(W\left(G_{1}\right)-3\left\|G_{1}\right\|+\left|G_{1}\right|\left(\left|G_{1}\right|-1\right)\right)}\right] \\
& \quad+\left\|G_{1}\right\|\left(1+\frac{\left|G_{2}\right|}{2}+\frac{\left|G_{2}\right|^{2}}{3}\right)+\frac{1}{4}\left(\left|G_{2}\right|+3\right)\left|G_{1}\left\|G_{2}\left|+\frac{1}{2}\right| G_{1} \mid\right\| G_{2} \|\right.
\end{aligned}
$$

with equality holding if and only if $G_{1}$ is isomorphic to a graph of diameter 2.

Proof Using Corollaries 1 and 2 with Theorem 2, from Theorem 4, we get the lower and upper bounds on the Harary index of the corona product. Moreover, the equality holds if and only if $G_{1}$ is isomorphic to a graph of diameter 2 .

Given a graph $G$ with vertex set $V=\left\{v_{1}, v_{2}, \ldots, v_{n}\right\}$, the thorn graph $G\left(p_{1}, p_{2}, \ldots, p_{n}\right)$ first introduced by Gutman [24], is a graph obtained by attaching $p_{i}$ pendent vertices to vertex $v_{i}$ for $i=1,2, \ldots, n$. In particular, if $p_{1}=p_{2}=\cdots=p_{n}=p$, we denote by $G^{(p)}$ the thorn graph $G\left(p_{1}, p_{2}, \ldots, p_{n}\right)$ for short. Recall the definition of the corona product, the graph $G^{(p)} \cong G \circ \overline{K_{p}}$, where $\overline{K_{p}}$ denotes the complement of a complete graph $K_{p}$. Therefore, for a connected graph $G$ of order $n$, we have the following.

\section{Example 2}

$$
H\left(G^{(p)}\right)=H\left(G \circ \overline{K_{p}}\right)=H(G)+p H_{1}(G)+p^{2} H_{2}\left(G_{1}\right)+\frac{1}{4}(p+3) n p .
$$

The Cartesian product $G_{1} \times G_{2}$ of graphs $G_{1}$ and $G_{2}$ has the vertex set $V\left(G_{1} \times G_{2}\right)=$ $V\left(G_{1}\right) \times V\left(G_{2}\right)$ and $\left(u_{i}, v_{j}\right)\left(u_{k}, v_{\ell}\right)$ is an edge of $G_{1} \times G_{2}$ if $u_{i}=u_{k}$ and $v_{j} v_{\ell} \in E\left(G_{2}\right)$, or $u_{i} u_{k} \in E\left(G_{1}\right)$ and $v_{j}=v_{\ell}$.

Theorem 6 Let $G_{1}$ and $G_{2}$ be two connected graphs with diameter $D\left(G_{2}\right)$ of the graph $G_{2}$.

Then

$$
\begin{aligned}
& \left|G_{1}\right| H\left(G_{2}\right)+\left|G_{2}\right| H\left(G_{1}\right)+\left|G_{2}\right|\left(\left|G_{2}\right|-1\right) H_{D}\left(G_{1}\right) \\
& \quad \leq H\left(G_{1} \times G_{2}\right) \\
& \quad \leq\left|G_{1}\right| H\left(G_{2}\right)+\left|G_{2}\right| H\left(G_{1}\right)+\left|G_{2}\right|\left(\left|G_{2}\right|-1\right) H_{1}\left(G_{1}\right),
\end{aligned}
$$

where

$$
\begin{aligned}
& H_{D}\left(G_{1}\right)=\sum_{u_{i}, u_{k} \in V\left(G_{1}\right), i \neq k} \frac{1}{d_{G_{1}}\left(u_{i}, u_{k}\right)+D\left(G_{2}\right)} \text { and } \\
& H_{1}\left(G_{1}\right)=\sum_{u_{i}, u_{k} \in V\left(G_{1}\right), i \neq k} \frac{1}{d_{G_{1}}\left(u_{i}, u_{k}\right)+1} .
\end{aligned}
$$


Moreover, both sides of the equality hold in (8) if and only if $G_{2}$ is isomorphic to a complete graph of order $\left|G_{2}\right|$.

Proof By the definition of the Harary index, we have

$$
\begin{aligned}
& H\left(G_{1} \times G_{2}\right)=\sum_{\left(u_{i}, v_{j}\right),\left(u_{k}, v_{\ell}\right) \in V\left(G_{1} \times G_{2}\right),\left(u_{i}, v_{j}\right) \neq\left(u_{k}, v_{\ell}\right)} \frac{1}{d_{G_{1} \times G_{2}}\left(\left(u_{i}, v_{j}\right),\left(u_{k}, v_{\ell}\right)\right)} \\
& =\sum_{\left(u_{i}, v_{j}\right),\left(u_{k}, v_{\ell}\right) \in V\left(G_{1} \times G_{2}\right), i=k, j \neq \ell} \frac{1}{d_{G_{1}}\left(u_{i}, u_{k}\right)+d_{G_{2}}\left(v_{j}, v_{\ell}\right)} \\
& +\sum_{\left(u_{i}, v_{j}\right),\left(u_{k}, v_{\ell}\right) \in V\left(G_{1} \times G_{2}\right), i \neq k, j=\ell} \frac{1}{d_{G_{1}}\left(u_{i}, u_{k}\right)+d_{G_{2}}\left(v_{j}, v_{\ell}\right)} \\
& +\sum_{\left(u_{i}, v_{j}\right),\left(u_{k}, v_{\ell}\right) \in V\left(G_{1} \times G_{2}\right), i \neq k, j \neq \ell} \frac{1}{d_{G_{1}}\left(u_{i}, u_{k}\right)+d_{G_{2}}\left(v_{j}, v_{\ell}\right)} \\
& =\sum_{u_{i} \in V\left(G_{1}\right)} \sum_{v_{j}, v_{\ell} \in V\left(G_{2}\right), j \neq \ell} \frac{1}{d_{G_{2}}\left(v_{j}, v_{\ell}\right)} \\
& +\sum_{u_{i}, u_{k} \in V\left(G_{1}\right), i \neq k} \sum_{v_{j} \in V\left(G_{2}\right)} \frac{1}{d_{G_{1}}\left(u_{i}, u_{k}\right)} \\
& +\sum_{u_{i}, u_{k} \in V\left(G_{1}\right), i \neq k} \sum_{v_{j}, v_{\ell} \in V\left(G_{2}\right), j \neq \ell} \frac{1}{d_{G_{1}}\left(u_{i}, u_{k}\right)+d_{G_{2}}\left(v_{j}, v_{\ell}\right)} \\
& \text { as } d_{G_{1}}\left(u_{i}, u_{i}\right)=0 \text { and } d_{G_{2}}\left(v_{j}, v_{j}\right)=0 \\
& \leq\left|G_{1}\right| H\left(G_{2}\right)+\left|G_{2}\right| H\left(G_{1}\right) \\
& +\sum_{\left(u_{i}, u_{k}\right) \in V\left(G_{1}\right)} \sum_{\left(v_{j}, v_{\ell}\right) \in V\left(G_{2}\right)} \frac{1}{d_{G_{1}}\left(u_{i}, u_{k}\right)+1} \\
& \text { as } d_{G_{2}}\left(v_{j}, v_{\ell}\right) \geq 1, j \neq \ell \\
& =\left|G_{1}\right| H\left(G_{2}\right)+\left|G_{2}\right| H\left(G_{1}\right)+\left|G_{2}\right|\left(\left|G_{2}\right|-1\right) H_{1}\left(G_{1}\right),
\end{aligned}
$$

where $H_{1}\left(G_{1}\right)$ is given in the statement of the theorem. Since $d_{G_{2}}\left(v_{j}, v_{\ell}\right) \leq D\left(G_{2}\right)$, similarly, we get

$$
\begin{aligned}
H\left(G_{1} \times G_{2}\right) \geq & \left|G_{1}\right| H\left(G_{2}\right)+\left|G_{2}\right| H\left(G_{1}\right) \\
& +\sum_{\left(u_{i}, u_{k}\right) \in V\left(G_{1}\right)} \sum_{\left(v_{j}, v_{\ell}\right) \in V\left(G_{2}\right)} \frac{1}{d_{G_{1}}\left(u_{i}, u_{k}\right)+D\left(G_{2}\right)} \\
= & \left|G_{1}\right| H\left(G_{2}\right)+\left|G_{2}\right| H\left(G_{1}\right)+\left|G_{2}\right|\left(\left|G_{2}\right|-1\right) H_{D}\left(G_{1}\right),
\end{aligned}
$$

where $H_{D}\left(G_{1}\right)$ is given in the statement of the theorem. The first part of the proof is over.

Suppose that both sides of the equality hold in (8). Then we must have $d_{G_{2}}\left(v_{j}, v_{\ell}\right)=1$, $v_{j} \neq v_{\ell}$, for all $v_{j}, v_{\ell} \in V\left(G_{2}\right)$ or $d_{G_{2}}\left(v_{j}, v_{\ell}\right)=D\left(G_{2}\right), v_{j} \neq v_{\ell}$, for all $v_{j}, v_{\ell} \in V\left(G_{2}\right)$. Hence $G_{2}$ is isomorphic to a complete graph of order $\left|G_{2}\right|$.

Conversely, one can see easily that (8) holds for $G_{2}$, a complete graph of order $\left|G_{2}\right|$. This completes the proof. 
Theorem 7 Let $G_{1}\left(¥ K_{\left|G_{1}\right|}\right)$ and $G_{2}\left(\not K_{\left|G_{2}\right|}\right)$ be two connected graphs with diameter $D\left(G_{1}\right)$ of the graph $G_{1}$ and $D\left(G_{2}\right)$ of the graph $G_{2}$. Then

$$
\begin{aligned}
H\left(G_{1} \times G_{2}\right)> & \left|G_{1}\right|\left\|G_{2}\right\|+\left|G_{2}\right|\left\|G_{1}\right\|+\frac{\left|G_{2}\right|\left(\left|G_{2}\right|-1\right)\left\|G_{1}\right\|}{D\left(G_{2}\right)+1}+\frac{\left(\frac{\left|G_{2}\right|\left(\left|G_{2}\right|-1\right)}{2}-\left\|G_{2}\right\|\right)^{2}\left|G_{1}\right|}{W\left(G_{2}\right)-\left\|G_{2}\right\|} \\
& +\left[\frac{\left|G_{2}\right|}{W\left(G_{1}\right)-\left\|G_{1}\right\|}+\frac{\left|G_{2}\right|\left(\left|G_{2}\right|-1\right)}{W\left(G_{1}\right)+\frac{\left|G_{1}\right|\left(\left|G_{1}\right|-1\right)}{2} D\left(G_{2}\right)-\left(D\left(G_{2}\right)+1\right)\left\|G_{1}\right\|}\right] \\
& \times\left(\frac{\left|G_{1}\right|\left(\left|G_{1}\right|-1\right)}{2}-\left\|G_{1}\right\|\right)^{2} .
\end{aligned}
$$

Moreover,

$$
\begin{aligned}
H\left(G_{1} \times G_{2}\right)< & \left|G_{1}\right||| G_{2}\left\|+\left|G_{2}\right|\right\| G_{1} \|+\frac{\left|G_{2}\right|\left(\left|G_{2}\right|-1\right)\left\|G_{1}\right\|}{2} \\
& +\frac{\left|G_{1}\right|\left(\frac{\left|G_{2}\right|\left(\left|G_{2}\right|-1\right)}{2}-\left\|G_{2}\right\|\right)\left[2+\left(\frac{\left|G_{2}\right|\left(\left|G_{2}\right|-1\right)}{2}-\left\|G_{2}\right\|\right) \times\left(\frac{D\left(G_{2}\right)}{2}+\frac{2}{D\left(G_{2}\right)}\right)\right]}{2\left(W\left(G_{2}\right)-\left\|G_{2}\right\|\right)} \\
& +\left[\frac{\left|G_{2}\right|\left(2+\left(\frac{\left|G_{1}\right| \mid\left(\left|G_{1}\right|-1\right)}{2}-\left\|G_{1}\right\|-1\right)\left(\frac{D\left(G_{1}\right)}{2}+\frac{2}{D\left(G_{1}\right)}\right)\right)}{2\left(W\left(G_{1}\right)-\left\|G_{1}\right\|\right)}\right. \\
& \left.+\frac{\left|G_{2}\right|\left(\left|G_{2}\right|-1\right)\left(2+\left(\frac{\left|G_{1}\right|\left(\left|G_{1}\right|-1\right)}{2}-\left\|G_{1}\right\|-1\right)\left(\frac{D\left(G_{1}\right)+1}{3}+\frac{3}{D\left(G_{1}\right)+1}\right)\right)}{2\left(W\left(G_{1}\right)+\frac{\left|G_{1}\right|\left(\left|G_{1}\right|-1\right)}{2}-2\left\|G_{1}\right\|\right)}\right] \\
& \times\left(\frac{\left|G_{1}\right|\left(\left|G_{1}\right|-1\right)}{2}-\left\|G_{1}\right\|\right) .
\end{aligned}
$$

Proof Using Theorems 1 and 2 with Corollary 1 in Theorem 6, we get the lower and upper bounds on the Cartesian product $G_{1} \times G_{2}$ of the graphs $G_{1}$ and $G_{2}$. Moreover, both inequalities are strict as $G_{1} \nsucceq K_{\left|G_{1}\right|}, G_{2} \nsucceq K_{\left|G_{2}\right|}$ and by Theorem 6 .

Theorem 8 Let $G_{1}$ and $G_{2}$ be two connected graphs with diameter $D\left(G_{1}\right)$ of the graph $G_{1}$.

Then

$$
\begin{aligned}
& \left|G_{1}\right| H\left(G_{2}\right)+\left|G_{2}\right| H\left(G_{1}\right)+\left|G_{1}\right|\left(\left|G_{1}\right|-1\right) H_{D}\left(G_{2}\right) \\
& \quad \leq H\left(G_{1} \times G_{2}\right) \\
& \quad \leq\left|G_{1}\right| H\left(G_{2}\right)+\left|G_{2}\right| H\left(G_{1}\right)+\left|G_{1}\right|\left(\left|G_{1}\right|-1\right) H_{1}\left(G_{2}\right),
\end{aligned}
$$

where

$$
\begin{aligned}
& H_{D}\left(G_{2}\right)=\sum_{v_{j}, v_{\ell} \in V\left(G_{2}\right), j \neq \ell} \frac{1}{d_{G_{2}}\left(v_{j}, v_{\ell}\right)+D\left(G_{1}\right)} \text { and } \\
& H_{1}\left(G_{2}\right)=\sum_{v_{j}, v_{\ell} \in V\left(G_{1}\right), j \neq \ell} \frac{1}{d_{G_{2}}\left(v_{j}, v_{\ell}\right)+1} .
\end{aligned}
$$

Moreover, both sides of the equality hold in (10) if and only if $G_{1}$ is isomorphic to a complete graph of order $\left|G_{1}\right|$. 
Proof Since $1 \leq d_{G_{1}}\left(u_{i}, u_{k}\right) \leq D\left(G_{1}\right), i \neq k$, from (9) we get the required result in (10). Moreover, both sides of the equality hold in (10) if and only if $G_{1}$ is isomorphic to a complete graph of order $\left|G_{1}\right|$.

Theorem 9 Let $G_{1}\left(¥ K_{\left|G_{1}\right|}\right)$ and $G_{2}\left(¥ K_{\left|G_{2}\right|}\right)$ be two connected graphs with diameter $D\left(G_{1}\right)$ of the graph $G_{1}$ and $D\left(G_{2}\right)$ of the graph $G_{2}$. Then

$$
\begin{aligned}
H\left(G_{1} \times G_{2}\right)> & \left|G_{1}\right|\left\|G_{2}\right\|+\left|G_{2}\right|\left\|G_{1}\right\|+\frac{\left|G_{1}\right|\left(\left|G_{1}\right|-1\right)\left\|G_{2}\right\|}{D\left(G_{1}\right)+1} \\
& +\frac{\left(\frac{\left|G_{1}\right|\left(\left|G_{1}\right|-1\right)}{2}-\left\|G_{1}\right\|\right)^{2}\left|G_{2}\right|}{W\left(G_{1}\right)-\left\|G_{1}\right\|} \\
& +\left[\frac{\left|G_{1}\right|}{W\left(G_{2}\right)-\left\|G_{2}\right\|}+\frac{\left|G_{1}\right|\left(\left|G_{1}\right|-1\right)}{W\left(G_{2}\right)+\frac{\left|G_{2}\right|\left(\left|G_{2}\right|-1\right)}{2} D\left(G_{1}\right)-\left(D\left(G_{1}\right)+1\right)\left\|G_{2}\right\|}\right] \\
& \times\left(\frac{\left|G_{2}\right|\left(\left|G_{2}\right|-1\right)}{2}-\left\|G_{2}\right\|\right)^{2} \cdot
\end{aligned}
$$

Moreover,

$$
\begin{aligned}
H\left(G_{1} \times G_{2}\right)< & \left|G_{1}\right|\left\|G_{2}\right\|+\left|G_{2}\right|\left\|G_{1}\right\|+\frac{\left|G_{1}\right|\left(\left|G_{1}\right|-1\right)\left\|G_{2}\right\|}{2} \\
& +\frac{\left|G_{2}\right|\left(\frac{\left|G_{1}\right|\left(\left|G_{1}\right|-1\right)}{2}-\left\|G_{1}\right\|\right)\left[2+\left(\frac{\left|G_{1}\right|\left(\left|G_{1}\right|-1\right)}{2}-\left\|G_{1}\right\|\right) \times\left(\frac{D\left(G_{1}\right)}{2}+\frac{2}{D\left(G_{1}\right)}\right)\right]}{2\left(W\left(G_{1}\right)-\left\|G_{1}\right\|\right)} \\
& +\left[\frac{\left|G_{1}\right|\left(2+\left(\frac{\left|G_{2}\right|\left(\left|G_{2}\right|-1\right)}{2}-\left\|G_{2}\right\|-1\right)\left(\frac{D\left(G_{2}\right)}{2}+\frac{2}{D\left(G_{2}\right)}\right)\right)}{2\left(W\left(G_{2}\right)-\left\|G_{2}\right\|\right)}\right. \\
& \left.+\frac{\left|G_{1}\right|\left(\left|G_{1}\right|-1\right)\left(2+\left(\frac{\left|G_{2}\right|\left(\left|G_{2}\right|-1\right)}{2}-\left\|G_{2}\right\|-1\right)\left(\frac{D\left(G_{2}\right)+1}{3}+\frac{3}{D\left(G_{2}\right)+1}\right)\right)}{2\left(W\left(G_{2}\right)+\frac{\left|G_{2}\right|\left(\left|G_{2}\right|-1\right)}{2}-2\left\|G_{2}\right\|\right)}\right] \\
& \times\left(\frac{\left|G_{2}\right|\left(\left|G_{2}\right|-1\right)}{2}-\left\|G_{2}\right\|\right) .
\end{aligned}
$$

Proof Using Theorems 1 and 2 with Corollary 1 in Theorem 8, we get the lower and upper bound on the Cartesian product $G_{1} \times G_{2}$ of graphs $G_{1}$ and $G_{2}$. Moreover, both inequalities are strict as $G_{1} \nsucceq K_{\left|G_{1}\right|}, G_{2} \nsucceq K_{\left|G_{2}\right|}$ and by Theorem 8 .

Corollary 3 Let $G$ be a connected graph of order $|G|$. Then

$$
H\left(G \times K_{2}\right)=2 H(G)+|G|+2 H_{1}(G),
$$

where $H_{1}(G)=\sum_{v_{i}, v_{j} \in V(G), i \neq j} \frac{1}{d_{G}\left(v_{i}, v_{j}\right)+1}$.

Proof Choosing $G_{1}=G$ and $G_{2}=K_{2}$ in Theorem 6, this theorem follows immediately.

The lattice graph $L_{2, n}$ (see [25]) is just $P_{n} \times K_{2}$. It is well known that [2] $H\left(P_{n}\right)=n \sum_{i=1}^{n-1} \frac{1}{i}-$ $n+1$. So, we have the following example. 


\section{Example 3}

$$
\begin{aligned}
H\left(L_{2, n}\right) & =2 H\left(P_{n}\right)+n+2 H_{1}\left(P_{n}\right)=2 n \sum_{i=1}^{n-1} \frac{1}{i}-2 n+2+n+2 H_{1}\left(P_{n}\right) \\
& =4 n \sum_{i=3}^{n-1} \frac{1}{i}+n+6 .
\end{aligned}
$$

The composition (also called lexicographic product [26]) $G=G_{1}\left[G_{2}\right]$ of graphs $G_{1}$ and $G_{2}$ with disjoint vertex sets $V\left(G_{1}\right)$ and $V\left(G_{2}\right)$ and edge sets $E\left(G_{1}\right)$ and $E\left(G_{2}\right)$ is the graph with vertex set $V\left(G_{1}\right) \times V\left(G_{2}\right)$ and $\left(u_{i}, v_{j}\right)$ is adjacent with $\left(u_{k}, v_{\ell}\right)$ whenever $u_{i}$ is adjacent with $u_{k}$, or $u_{i}=u_{k}$ and $v_{j}$ is adjacent with $v_{\ell}$.

Theorem 10 Let $G_{1}$ and $G_{2}$ be two connected graphs. Then

$$
H\left(G_{1}\left[G_{2}\right]\right)=\frac{1}{4}\left|G_{1}\right|\left|G_{2}\right|\left(\left|G_{2}\right|-1\right)+\frac{1}{2}\left|G_{1}\right|\left\|G_{2}\right\|+\left|G_{2}\right|^{2} H\left(G_{1}\right) .
$$

Proof By the definition of the Harary index, we have

$$
\begin{aligned}
& H\left(G_{1}\left[G_{2}\right]\right)=\sum_{\left(u_{i}, v_{j}\right),\left(u_{k}, v_{\ell}\right) \in V\left(G_{1}\left[G_{2}\right]\right),\left(u_{i}, v_{j}\right) \neq\left(u_{k}, v_{\ell}\right)} \frac{1}{d_{G_{1}\left[G_{2}\right]}\left(\left(u_{i}, v_{j}\right),\left(u_{k}, v_{\ell}\right)\right)} \\
& =\sum_{\left(u_{i}, v_{j}\right),\left(u_{i}, v_{\ell}\right) \in V\left(G_{1}\left[G_{2}\right], j \neq \ell\right.} \frac{1}{d_{G_{1}\left[G_{2}\right]}\left(\left(u_{i}, v_{j}\right),\left(u_{i}, v_{\ell}\right)\right)} \\
& +\sum_{\left(u_{i}, v_{j}\right),\left(u_{k}, v_{\ell}\right) \in V\left(G_{1}\left[G_{2}\right], i \neq k\right.} \frac{1}{d_{G_{1}\left[G_{2}\right]}\left(\left(u_{i}, v_{j}\right),\left(u_{k}, v_{\ell}\right)\right)} \\
& =\sum_{u_{i} \in V\left(G_{1}\right)} \frac{1}{2} \sum_{j=1}^{\left|G_{2}\right|}\left[d_{G_{2}}\left(v_{j}\right)+\frac{1}{2}\left(\left|G_{2}\right|-d_{G_{2}}\left(v_{j}\right)-1\right)\right] \\
& +\sum_{v_{j} \in V\left(G_{2}\right)} \sum_{u_{i}, u_{k} \in V\left(G_{1}\right), i \neq k} \frac{\left|G_{2}\right|}{d_{G_{1}}\left(u_{i}, u_{k}\right)} \\
& \text { as } d_{G_{1}\left[G_{2}\right]}\left(\left(u_{i}, v_{j}\right),\left(u_{i}, v_{\ell}\right)\right)= \begin{cases}0 & \text { if } v_{j}=v_{\ell}, \\
1 & \text { if } v_{j} v_{\ell} \in E\left(G_{2}\right), \\
2 & \text { otherwise }\end{cases} \\
& =\frac{\left|G_{1}\right|}{4} \sum_{j=1}^{\left|G_{2}\right|}\left(\left|G_{2}\right|+d_{G_{2}}\left(v_{j}\right)-1\right)+\left|G_{2}\right|^{2} \sum_{u_{i}, u_{k} \in V\left(G_{1}\right), i \neq k} \frac{1}{d_{G_{1}}\left(u_{i}, u_{k}\right)} \\
& =\frac{1}{4}\left|G_{1}\right|\left|G_{2}\right|\left(\left|G_{2}\right|-1\right)+\left.\frac{1}{2}\left|G_{1}\right|\left|G_{2} \|+\right| G_{2}\right|^{2} H\left(G_{1}\right) .
\end{aligned}
$$

The double graph of a given graph $G$, denoted by $G^{\circledast}$, is constructed by making two copies of $G$ (including the initial edge set of each), denoted by $G_{1}$ and $G_{2}$, and adding edges $u_{1} v_{2}$ and $u_{2} v_{1}$ for every edge $u v$ of $G$. From the definition of composition, we conclude that $G^{\circledast} \cong G\left[K_{2}\right]$ for any connected graph $G$. Therefore the following corollary can be easily obtained. 
Corollary 4 Let $G$ be a connected graph. Then

$$
H\left(G\left[K_{2}\right]\right)=\frac{3|G|}{2}+4 H(G)
$$

For a complete graph $K_{n}$, we find that $K_{n}^{\circledast}$ is a graph obtained by deleting a perfect matching from the complete graph $K_{2 n}$, which is just the well-known cocktail party graph (see [27]).

\section{Example 4}

$$
H\left(K_{n}^{\circledast}\right)=\frac{3 n}{2}+4 H\left(K_{n}\right)=2 n^{2}-\frac{n}{2} .
$$

The disjunction $G_{1} \otimes G_{2}$ of graphs $G_{1}$ and $G_{2}$ is the graph with vertex set $V\left(G_{1}\right) \times V\left(G_{2}\right)$ and $\left(u_{i}, v_{j}\right)$ is adjacent with $\left(u_{k}, v_{\ell}\right)$ whenever $u_{i} u_{k} \in E\left(G_{1}\right)$ or $v_{j} v_{\ell} \in E\left(G_{2}\right)$.

Theorem 11 Let $G_{1}$ and $G_{2}$ be two connected graphs. Then

$$
H\left(G_{1} \otimes G_{2}\right)=\frac{1}{4}\left|G_{1}\right|\left|G_{2}\right|\left(\left|G_{1} \| G_{2}\right|-1\right)+\frac{1}{2}\left(\left\|G_{1}\right\|\left|G_{2}\right|^{2}+\left\|G_{2}\right\|\left|G_{1}\right|^{2}\right)-\left\|G_{1}\right\|\left\|G_{2}\right\| .
$$

Proof In [22], it has been proved that

$$
d_{G_{1} \otimes G_{2}}\left(\left(u_{i}, v_{j}\right),\left(u_{k}, v_{\ell}\right)\right)= \begin{cases}0 & u_{i}=u_{k} \text { and } v_{j}=v_{\ell}, \\ 1 & u_{i} u_{k} \in E(G) \text { or } v_{j} v_{\ell} \in E(H), \\ 2 & \text { otherwise. }\end{cases}
$$

Moreover, it has been showed that

$$
\begin{aligned}
& \left|\left\{v \in V\left(G_{1} \otimes G_{2}\right) \mid d_{G_{1} \otimes G_{2}}\left(\left(u_{i}, v_{j}\right), v\right)=1\right\}\right| \\
& \quad=d_{G_{1}}\left(u_{i}\right)\left|G_{2}\right|+d_{G_{2}}\left(v_{j}\right)\left|G_{1}\right|-d_{G_{1}}\left(u_{i}\right) d_{G_{2}}\left(v_{j}\right) .
\end{aligned}
$$

By the definition of the Harary index, we have

$$
\begin{aligned}
H\left(G_{1} \otimes G_{2}\right)= & \sum_{\left(u_{i}, v_{j}\right),\left(u_{k}, v_{\ell}\right) \in V\left(G_{1} \otimes G_{2}\right),\left(u_{i}, v_{j}\right) \neq\left(u_{k}, v_{\ell}\right)} \frac{1}{d_{G_{1} \otimes G_{2}}\left(\left(u_{i}, v_{j}\right),\left(u_{k}, v_{\ell}\right)\right)} \\
= & \frac{1}{2} \sum_{\left(u_{i}, v_{j}\right) \in V\left(G_{1} \otimes G_{2}\right)}\left[d_{G_{1} \otimes G_{2}}\left(\left(u_{i}, v_{j}\right)\right)+\frac{1}{2}\left(\left|G_{1}\right|\left|G_{2}\right|-d_{G_{1} \otimes G_{2}}\left(\left(u_{i}, v_{j}\right)\right)-1\right)\right] \\
& \text { by }(11) \\
= & \frac{1}{4}\left|G_{1}\right|\left|G_{2}\right|\left(\left|G_{1}\right|\left|G_{2}\right|-1\right) \\
& +\frac{1}{4} \sum_{\left(u_{i}, v_{j}\right) \in V\left(G_{1} \otimes G_{2}\right)}\left(d_{G_{1}}\left(u_{i}\right)\left|G_{2}\right|+d_{G_{2}}\left(v_{j}\right)\left|G_{1}\right|-d_{G_{1}}\left(u_{i}\right) d_{G_{2}}\left(v_{j}\right)\right) \quad \text { by }(12) \\
= & \frac{1}{4}\left|G_{1}\right|\left|G_{2}\right|\left(\left|G_{1}\right|\left|G_{2}\right|-1\right)
\end{aligned}
$$




$$
\begin{aligned}
& +\frac{1}{4} \sum_{u_{i} \in V\left(G_{1}\right)} \sum_{v_{j} \in V\left(G_{2}\right)}\left(d_{G_{1}}\left(u_{i}\right)\left|G_{2}\right|+d_{G_{2}}\left(v_{j}\right)\left|G_{1}\right|-d_{G_{1}}\left(u_{i}\right) d_{G_{2}}\left(v_{j}\right)\right) \\
= & \frac{1}{4}\left|G_{1}\right|\left|G_{2}\right|\left(\left|G_{1}\right|\left|G_{2}\right|-1\right)+\frac{1}{2}\left(\left\|G_{1}\right\|\left|G_{2}\right|^{2}+\left\|G_{2}\right\|\left|G_{1}\right|^{2}\right)-\left\|G_{1}\right\|\left\|G_{2}\right\| .
\end{aligned}
$$

The construction of the extended double cover was introduced by Alon [28] in 1986. For a simple graph $G$ with vertex set $V=\left\{v_{1}, v_{2}, \ldots, v_{n}\right\}$, the extended double cover of $G$, denoted by $G^{\star}$, is the bipartite graph with bipartition $(X ; Y)$ where $X=\left\{x_{1}, x_{2}, \ldots, x_{n}\right\}$ and $Y=\left\{y_{1}, y_{2}, \ldots, y_{n}\right\}$, in which $x_{i}$ and $y_{j}$ are adjacent if and only if $i=j$ or $v_{i}$ and $v_{j}$ are adjacent in $G$. Note that for a graph $G, G^{\star} \cong G \otimes K_{2}$. So, the corollary below follows immediately.

Corollary 5 Let $G$ be a connected graph of order $n$. Then

$$
H\left(G \otimes K_{2}\right)=\frac{1}{2} n(2 n-1)+\frac{1}{2}\left(4\|G\|+n^{2}\right)-\|G\| .
$$

For a complete graph $K_{n}$, by the definition listed above, we find that $K_{n} \otimes K_{2}$ is just $K_{n, n}$.

\section{Example 5}

$$
H\left(K_{n} \otimes K_{2}\right)=\frac{1}{2} n(2 n-1)+\frac{1}{2}\left(4\left\|K_{n}\right\|+n^{2}\right)-\left\|K_{n}\right\|=2 n^{2}-n .
$$

Let $G=(V, E)$ be a connected graph of $|G|$ vertices with $\|G\|$ edges. If we put two similar graphs $G$ side by side, and any vertex of the first graph $G$ is connected by edges with those vertices which are adjacent to the corresponding vertex of the second graph $G$ and the resultant graph is denoted by $K_{2} \uplus G$, then we have $\left|K_{2} \uplus G\right|=2|G|$ and $\left\|K_{2} \uplus G\right\|=\|G\|+$ $\|G\|+2\|G\|=4\|G\|$. Moreover, $K_{2} \uplus G$ is the graph of $K_{2}$ and $G$ with the vertex set $V\left(K_{2} \times\right.$ $G)=V\left(K_{2}\right) \times V(G)$ and $\left(u_{i}, v_{j}\right)\left(u_{k}, v_{\ell}\right)$ is an edge of $K_{2} \times G$ whenever $\left(u_{i}=u_{k}\right.$ and $v_{j}$ is adjacent with $\left.v_{\ell}\right)$ or $\left(u_{i} \neq u_{k}\right.$ and $v_{j}$ is adjacent with $\left.v_{\ell}\right)$.

\section{Theorem 12 Let $G$ be a connected graph. Then}

$$
H\left(K_{2} \uplus G\right)=4 H(G)+\frac{|G|}{2} .
$$

Proof By the definition of the Harary index, we have

$$
\begin{aligned}
H\left(K_{2} \uplus G\right)= & \sum_{\left(u_{i}, v_{j}\right),\left(u_{k}, v_{\ell}\right) \in V\left(K_{2} \uplus G_{2}\right),\left(u_{i}, v_{j}\right) \neq\left(u_{k}, v_{\ell}\right)} \frac{1}{d_{K_{2} \uplus G}\left(\left(u_{i}, v_{j}\right),\left(u_{k}, v_{\ell}\right)\right)} \\
= & \sum_{\left(u_{i}, v_{j}\right),\left(u_{i}, v_{\ell}\right) \in V\left(K_{2} \uplus G\right), j \neq \ell} \frac{1}{d_{K_{2} \uplus G}\left(\left(u_{i}, v_{j}\right),\left(u_{i}, v_{\ell}\right)\right)} \\
& +\sum_{\left(u_{i}, v_{j}\right),\left(u_{k}, v_{\ell}\right) \in V\left(K_{2} \uplus G\right), i \neq k} \frac{1}{d_{K_{2} \uplus G}\left(\left(u_{i}, v_{j}\right),\left(u_{k}, v_{\ell}\right)\right)} \\
= & \sum_{u_{i} \in K_{2}} \sum_{v_{j}, v_{\ell} \in V(G), j \neq \ell} \frac{1}{d_{G}\left(v_{j}, v_{\ell}\right)}+\sum_{v_{j} \in V(G)}\left[\frac{1}{2}+\sum_{v_{\ell} \in V(G), \ell \neq j} \frac{1}{d_{G}\left(v_{j}, v_{\ell}\right)}\right] \\
= & 2 \sum_{v_{j}, v_{\ell} \in V(G), j \neq \ell} \frac{1}{d_{G}\left(v_{j}, v_{\ell}\right)}+\frac{|G|}{2}+\sum_{v_{j} \in V(G)} \sum_{v_{\ell} \in V(G), \ell \neq j} \frac{1}{d_{G}\left(v_{j}, v_{\ell}\right)}
\end{aligned}
$$




$$
\begin{aligned}
= & 4 H(G)+\frac{|G|}{2} \\
& \text { as } H(G)=\sum_{v_{j}, v_{\ell} \in V(G), \ell \neq j} \frac{1}{d_{G}\left(v_{j}, v_{\ell}\right)}=\frac{1}{2} \sum_{v_{j} \in V(G)} \sum_{v_{\ell} \in V(G), \ell \neq j} \frac{1}{d_{G}\left(v_{j}, v_{\ell}\right)} .
\end{aligned}
$$

Let $G=(V, E)$ be a connected graph of $|G|$ vertices with $\|G\|$ edges. If we put two similar graphs $G$ side by side, and any vertex of the first graph $G$ is connected by edges with those vertices which are nonadjacent to the corresponding vertex (including the corresponding vertex itself) of the second graph $G$ and the resultant graph is denoted by $K_{2} \sqcup G$, then we have $\left|K_{2} \sqcup G\right|=2|G|$ and $\left\|K_{2} \sqcup G\right\|=\|G\|+\|G\|+|G|^{2}-2\|G\|=|G|^{2}$. Moreover, $K_{2} \sqcup G$ is the graph of $K_{2}$ and $G$ with the vertex set $V\left(K_{2} \sqcup G\right)=V\left(K_{2}\right) \times V(G)$ and $\left(u_{i}, v_{j}\right)\left(u_{k}, v_{\ell}\right)$ is an edge of $K_{2} \times G$ whenever $\left(u_{i}=u_{k}\right.$ and $v_{j}$ is adjacent with $\left.v_{\ell}\right)$ or $\left(u_{i} \neq u_{k}\right.$ and $v_{j}$ is nonadjacent with $v_{\ell}$ ).

Theorem 13 Let $G$ be a connected graph of order $|G|$. Then

$$
H\left(K_{2} \sqcup G\right)=\frac{|G|(3|G|-1)}{2} .
$$

Proof In $K_{2} \sqcup G$, for each vertex $\left(u_{i}, v_{j}\right)$, there are $d_{G}\left(v_{j}\right)+|G|-1-d_{G}\left(v_{j}\right)+1=|G|$ neighbors, and $d_{G}\left(v_{j}\right)+n-1-d_{G}\left(v_{j}\right)=|G|-1$ vertices with the distance 2 from itself. By the definition of the Harary index, we have

$$
\begin{aligned}
H\left(K_{2} \sqcup G\right) & =\sum_{\left(u_{i}, v_{j}\right),\left(u_{k}, v_{\ell}\right) \in V\left(K_{2} \sqcup G_{2}\right),\left(u_{i}, v_{j}\right) \neq\left(u_{k}, v_{\ell}\right)} \frac{1}{d_{K_{2} \sqcup G}\left(\left(u_{i}, v_{j}\right),\left(u_{k}, v_{\ell}\right)\right)} \\
& =\sum_{u_{i} \in K_{2}} \frac{1}{2} \sum_{v_{j} \in G}\left(d_{K_{2} \sqcup G}\left(u_{i}, v_{j}\right)+\frac{2|G|-d_{K_{2} \sqcup G}\left(u_{i}, v_{j}\right)-1}{2}\right) \\
& =\sum_{u_{i} \in K_{2}} \frac{1}{2} \sum_{v_{j} \in G}\left(|G|+\frac{|G|-1}{2}\right) \\
& =\frac{|G|(3|G|-1)}{2} .
\end{aligned}
$$

\section{Competing interests}

The authors declare that they have no competing interests.

\section{Authors' contributions}

All authors completed the paper together. All authors read and approved the final manuscript.

\section{Author details}

'Department of Mathematics, Sungkyunkwan University, Suwon, 440-746, Republic of Korea. ${ }^{2}$ College of Science, Nanjing University of Aeronautics \& Astronautics, Nanjing, Jiangsu, P.R. China. ${ }^{3}$ Department of Mathematics, Faculty of Arts and Science, Uludag University, Gorukle Campus, Bursa, 16059, Turkey. ${ }^{4}$ Department of Mathematics, Faculty of Science, Selçuk University, Campus, Konya, 42075, Turkey. ${ }^{5}$ Faculty of Science, University of Split, Nikole Tesle 12, Split, HR-21000, Croatia.

\section{Acknowledgements}

KCD was supported by the Faculty research Fund, Sungkyunkwan University, 2012, and National Research Foundation funded by the Korean government with the grant no. 2013R1A1A2009341; and KX was supported by China Postdoctoral Science Foundation (2013M530253) and the NNSF of China with the number 11201227. Moreover, INC and ASC were supported by the Scientific Project Office Funds (BAP) of Uludag (with Project Code F2012/15, F2012/19 and F2012/20) and Selcuk (with Project Code 13701071) Universities, respectively. 


\section{References}

1. Ivanciuc, O, Balaban, TS, Balaban, AT: Reciprocal distance matrix, related local vertex invariants and topological indices. J. Math. Chem. 12, 309-318 (1993)

2. Plavšić, D, Nikolić, S, Trinajstić, N, Mihalić, Z: On the Harary index for the characterization of chemical graphs. J. Math. Chem. 12, 235-250 (1993)

3. Das, KC, Zhou, B, Trinajstić, N: Bounds on Harary index. J. Math. Chem. 46, 1369-1376 (2009)

4. Das, KC, Xu, K, Gutman, I: On Zagreb and Harary indices. MATCH Commun. Math. Comput. Chem. 70(1), 301-314 (2013)

5. Ilić, A, Yu, G, Feng, L: The Harary index of trees. Util. Math. 87, 21-32 (2012)

6. Lučić, B, Miličević, A, Nikolić, S, Trinajstić, N: Harary index - twelve years later. Croat. Chem. Acta 75, 847-868 (2002)

7. Xu, K: Trees with the seven smallest and eight greatest Harary indices. Discrete Appl. Math. 160, 321-331 (2012)

8. Xu, K, Das, KC: Extremal unicyclic and bicyclic graphs with respect to Harary index. Bull. Malays. Math. Soc. 36(2), 373-383 (2013)

9. Xu, K, Das, KC: On Harary index of graphs. Discrete Appl. Math. 159, 1631-1640 (2011)

10. Xu, K, Trinajstić, N: Hyper-Wiener and Harary indices of graphs with cut edges. Util. Math. 84, 153-163 (2011)

11. Zhou, B, Cai, X, Trinajstić, N: On the Harary index. J. Math. Chem. 44, 611-618 (2008)

12. Wiener, H: Structural determination of paraffin boiling points. J. Am. Chem. Soc. 69, 17-20 (1947)

13. Gutman, I, Polansky, OE: Mathematical Concepts in Organic Chemistry. Springer, Berlin (1986)

14. Todeschini, R, Consonni, V: Handbook of Molecular Descriptors. Wiley, Weinheim (2000)

15. Needham, DE, Wei, IC, Seybold, PG: Molecular modeling of the physical properties of alkanes. J. Am. Chem. Soc. 110, 4186-4194 (1988)

16. Rücker, G, Rücker, C: On topological indices, boiling points, and cycloalkanes. J. Chem. Inf. Comput. Sci. 39, 788-802 (1999)

17. Das, KC, Gutman, I: Estimating the Wiener index by means of number of vertices, number of edges, and diameter. MATCH Commun. Math. Comput. Chem. 64(3), 647-660 (2010)

18. Dobrynin, AA, Entringer, R, Gutman, I: Wiener index of trees: theory and applications. Acta Appl. Math. 66, 211-249 (2001)

19. Liu, H, Pan, XF: On the Wiener index of trees with fixed diameter. MATCH Commun. Math. Comput. Chem. 60, 85-94 (2008)

20. Zhang, XD, Xiang, QY, Xu, LQ, Pan, RY: The Wiener index of trees with given degree sequence. MATCH Commun. Math Comput. Chem. 60, 623-644 (2008)

21. Bondy, JA, Murty, USR: Graph Theory with Applications. Macmillan Press, New York (1976)

22. Khalifeh, MH, Azari, HY, Ashrafi, AR: The hyper-Wiener index of graph operations. Comput. Math. Appl. 56, 1402-1407 (2008)

23. Imrich, W, Klavžar, S: Product Graphs: Structure and Recognition. Wiley, New York (2000)

24. Gutman, I: Distance in theory of graphs. Publ. Inst. Math. (Belgr.) 63, 31-36 (1998)

25. Brouwer, AE, Cohen, AM, Neumaier, A: Distance-Regular Graphs. Springer, Berlin (1989)

26. Harary, F: Graph Theory. Addison-Wesley, Reading (1994) p. 22

27. Biggs, NL: Algebraic Graph Theory, 2nd edn. Cambridge University Press, Cambridge (1993) pp. 17 and 68

28. Alon, N: Eigenvalues and expanders. Combinatorica 6, 83-96 (1986)

\section{Submit your manuscript to a SpringerOpen ${ }^{\circ}$ journal and benefit from:}

- Convenient online submission

- Rigorous peer review

- Immediate publication on acceptance

- Open access: articles freely available online

- High visibility within the field

- Retaining the copyright to your article 